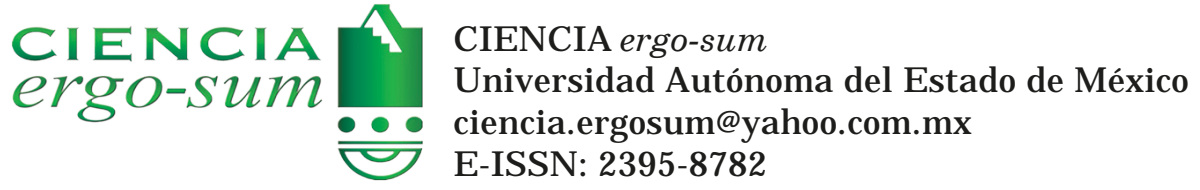

\title{
Evaluación de la política de seguridad pública en México: 2006-2018
}

Ramírez González, Karen

Evaluación de la política de seguridad pública en México: 2006-2018

CIE NCIA ergo-sum, vol. 28, núm. 2, julio-octubre 2021|e119

Universidad Autónoma del Estado de México, México

Esta obra está bajo una Licencia Creative Commons Atribución-NoComercial-SinDerivar 4.0 Internacional .

Ramírez González, K. (2021). Evaluación de la política de seguridad pública en México: 2006-2018. CI ENCIA ergo-sum, 28(2). https://doi.org/10.30878/ces.v28n2a2 


\title{
Evaluación de la política de seguridad pública en México: 2006-2018 Evaluation of public security policy in Mexico: 2006-2018
}

\author{
Karen Ramírez González \\ El Colegio de Morelos, México \\ karenrg830704@hotmail.com \\ https://orcid.org/0000-0003-0279-0491
}

Recepción: 10 de octubre de 2019

Aprobación: 24 de febrero de 2021

\begin{abstract}
RESUMEN
El artículo evalúa la política de seguridad pública en México durante los sexenios de Felipe Calderón Hinojosa y Enrique Peña Nieto, a partir de los resultados de la ENVIPE, desde su primera publicación en 2010 hasta 2018. El análisis considera tres líneas sustanciales: la percepción de la inseguridad, la confianza institucional y la valoración ciudadana sobre el desempeño gubernamental para reducir la inseguridad en el país. Para obtener los resultados, se realizó una evaluación de tipo externa y ex post facto, de la cual se concluyó que la valoración social sobre la intervención gubernamental para el mejoramiento de la seguridad pública resultó reprobatoria y prácticamente imperceptible para la ciudadanía durante los dos sexenios.
\end{abstract}

Palabras clave: percepción de la inseguridad, confianza institucional, condición de victimización, evaluación, política pública.

\section{Abstract}

The article evaluates the public security policy in Mexico during the six-year terms of Felipe Calderón Hinojosa and Enrique Peña Nieto, based on the results of ENVIPE, from its first publication in 2010 to 2018. The analysis considers three substantial lines: the perception of insecurity, institutional trust, and the citizen's assessment of government performance to reduce insecurity in the country. To obtain the results, an external and ex-post-facto evaluation was carried out, from which it was concluded that the social assessment of the government intervention for the improvement of public security was reprobative and practically imperceptible to citizens, during both six-year periods.

KEYWORDS: perception of insecurity, institutional trust, victimization status, evaluation, public policy.

\section{INTRODUCCIÓN}

La evaluación de las políticas públicas en México es un tema que comienza a tomar interés en la academia y en los gobiernos, principalmente federales, desde el año 2000. El desarrollo a nivel federal es más notable porque programar, medir y detectar fallos, requiere de una base de datos confiable en materia de diseño e implementación de políticas públicas, servicios, programas o proyectos que puedan ser medidos a través de indicadores de gestión y de resultados (por mencionar algunos), además del apoyo técnico de personal especializado, la disponibilidad de las instituciones para transparentar la información, la cooperación del personal involucrado, o de aquellos que tengan algún tipo de interés concerniente a la política pública (stakeholders), y que permitan ser considerados como parte del proceso evaluativo.

La evaluación no sólo se remite al logro de los objetivos programados por las instituciones, sino también, a la percepción de los beneficiarios de la intervención gubernamental, en este caso, la ciudadanía. Es un proceso integral que debe ser constantemente medido (ex ante y ex post facto). Requiere de personal multidisciplinario que sea sensible a los cambios de aquello que será objeto de evaluación (el evaluando).

La seguridad pública es una función compleja de estudiar y evaluar debido a que la gran mayoría de los datos forman parte de las líneas de acción implementadas por el gobierno y que se consideran como "confidenciales", además de la opacidad de la información reportada por las instituciones oficiales, las cuales a menudo no 
concuerdan con la evidencia recabada por las asociaciones civiles o, en varios de los casos, no se lleva un conteo específico sobre los recursos disponibles, gastados y solicitados, por lo que se dificulta el trabajo de los evaluadores.

Pese a las complejidades, ha habido esfuerzos notorios por estudiar la seguridad pública, donde destacan cuatro vertientes. La primera se centra en estudios de caso locales, como las investigaciones de Laca y Navarro (2013), Becerra y Trujano (2011), Sánchez y Aguilar (2016) y Dzib et al. (2016). La segunda vertiente aborda el impacto de la inseguridad en la democracia. Las investigaciones más relevantes son aquellas elaboradas por Ruiz y Turcios (2009), Gómez (2012), Rodríguez (2012) y Alvarado (2010). Una tercera vertiente explora los efectos de la violencia en lo económico. Las investigaciones de Soria $(2017,2018)$ y Rebolledo $(2015)$ son pioneras en este campo escasamente explorado. Una cuarta línea indaga las causas y las consecuencias de la violencia en la percepción social y en la vida cotidiana. En esta vertiente son representativos los estudios de García (2012), Tapia (2013), Chávez y Esparza (2017).

De ahí que el título de esta disertación trate de contribuir a esclarecer que la seguridad pública tiene un enfoque estadocéntrico, el cual implica salvaguardar la vida, la propiedad y la libertad de los ciudadanos por medio de estrategias diseñadas por las élites estatales. Abarca en primer lugar la seguridad nacional, entendida como el resguardo a la soberanía del país, así como la prevención y combate de los riesgos potenciales que pongan en peligro la existencia de la nación, y, en segundo término, la seguridad interior, que está enfocada en el buen funcionamiento de las instituciones, el mejoramiento de la convivencia de los ciudadanos, la prevención y sanción del delito del fuero común, entre otros aspectos. Por ello, se propone en este análisis, la evaluación de la política de seguridad como un componente global y no como acciones estratégicas o sectorizadas.

Para los fines metodológicos perseguidos, las encuestas realizadas por el Estado son herramientas de fácil acceso para medir el impacto nacional de la seguridad pública. En primer lugar, por la capacidad de abarcamiento del muestreo y, en segundo lugar, por el diseño de las preguntas que tratan de reflejar la valoración social tanto de la seguridad nacional como de la seguridad interior.

Este trabajo se distingue de otros porque se propone como indicador de evaluación, de la efectividad de la política de seguridad pública durante el sexenio de Felipe Calderón Hinojosa y Enrique Peña Nieto, que es medida por la Encuesta Nacional de Victimización y Percepción sobre Seguridad Pública (ENVIPE). Lo anterior es el principal aporte de este trabajo, ya que una gran mayoría de las investigaciones que han analizado los resultados de la Encuesta consideran uno o dos años de análisis, y se centran en la percepción social y en el imaginario colectivo. La segunda contribución consiste en estudiar los resultados históricos a partir de la primera publicación en 2010 hasta 2018. En tercer lugar, se pretende mostrar un estimado de la evolución de los delitos en México en el sexenio actual.

El objetivo general de este artículo consiste en evaluar la efectividad de la política de seguridad pública mediante la percepción de la seguridad y la confianza institucional, así como el desempeño gubernamental a través de acciones específicas para su mejora que son visibles para la población y, en concreto, la función de los cuerpos policiales. Como objetivos específicos, se analiza la evolución de los delitos como porcentaje de la población total en México, considerando las denuncias y la denominada cifra negra, y se muestran las perspectivas de las variaciones en los delitos para el sexenio actual.

La pregunta que se tratará de responder es la siguiente: ¿los logros reportados durante el sexenio de Felipe Calderón Hinojosa y Enrique Peña Nieto corresponden con la valoración de la ciudadanía? La hipótesis que subyace ante esta interrogante es la siguiente: a pesar de la reducción de delitos como el secuestro y el homicidio, la percepción negativa de la seguridad pública continúa incrementándose desde 2010 a 2018 . En este trabajo se indagará cuáles son las posibles causas de esas concordancias o disonancias de la opinión pública..

Para lograr tales fines, el artículo se divide en cuatro secciones. La primera aborda el aspecto teórico y metodológico del diseño de este trabajo. La segunda analiza las estrategias generales de la política de seguridad en México durante los dos sexenios, así como las cifras reportadas en materia de delitos. La tercera explora los resultados de la ENVIPE hasta su última actualización efectuada el 24 de septiembre de 2019. Esta sección se subdivide 
en tres líneas generales: la percepción de la inseguridad, la confianza institucional y el conocimiento sobre la intervención pública. Finalmente, la cuarta sección realiza un estudio de prospectiva y enmarca los hallazgos principales de este estudio.

\section{Generalidades de la eValuación de POlíticas Públicas: una Propuesta Para la eValuación DE LA POLÍTICA DE SEGURIDAD PÚBLICA}

Con los albores de la alternancia partidista en el 2000, comenzaron a hacerse visibles los esfuerzos en materia de evaluación, en especial, para medir alcances y verificar que los presupuestos asignados a los programas públicos cumplieran con sus propósitos y, con ello, continuar financiándolos. Desafortunadamente, la evaluación en el ciclo de las políticas públicas es el proceso menos estudiado que otros. Cuando nos referimos a evaluar, se entiende como dar una apreciación o valoración cualitativa o cuantitativa a algo. Sin embargo, se le debe a Scrivent (1967) considerar la evaluación como un proceso científico, es decir, como la "ciencia de la valoración".

La evaluación es una actividad metodológica importante porque mejora los procesos, permite la rendición de cuentas y guía las decisiones para las acciones futuras (Olvera, 2013). Debido a que no es un proceso de culminación en el ciclo de las políticas, la evaluación puede realizarse en distintos procesos (Bustelo, 2001):

a) Según el papel del evaluador, puede ser de tipo formativa y sumativa. La primera es aplicable cuando se requiere mejorar el diseño de un programa mientras se va implementando. La segunda es acumulativa y ofrece una visión global de la política pública.

b) De acuerdo con el contenido o fase del programa, se clasifica en evaluación de diseño, procesos o resultados.

c) En cuanto al momento de la evaluación, puede ser ex ante (al inicio) o expost facto (durante la implementación o al final de la ejecución).

d) Conforme al agente evaluador, puede ser interna (cuando se realiza por parte de un equipo que trabaja en la institución) o externa (efectuada por un grupo de personas ajenas a la institución).

En este caso, la propuesta metodológica del artículo es realizar una evaluación ex post facto, ya que como políticas gubernamentales han concluido en 2012 y 2018, además de ser de tipo externa. Para tal efecto, se consideran los siguientes indicadores de medición:

a) Los resultados de la política de seguridad medidos en un primer indicador que muestra la proporción de las tasas de homicidios, secuestros, extorsiones, y un segundo indicador que cuantifica las tasas de los delitos cometidos, denunciados y la cifra negra.

b) La percepción de la inseguridad en el lugar de residencia por entidad, municipio o delegación y, colonia o localidad.

c) La tasa de victimización medida en porcentaje de población que considera ser víctima de al menos un delito, robo o asalto en la calle o transporte público, lesiones por agresión física, extorsión o secuestro. Este apartado se subdivide en la medición de las actividades, consideradas cotidianas, que la población dejó de realizar: salir de noche, permitir a sus hijos estar fuera de casa, portar dinero en efectivo o tarjetas de crédito o débito, y usar joyas.

d) El nivel de confianza institucional, contabilizada en el porcentaje de respuestas asignadas a las categorías sobre si la seguridad "mejorará", "seguirá igual de mal", "seguirá igual de bien" o "empeorará".

e) La percepción sobre la política pública y el desempeño policial. En este rubro se consideran diez instituciones de mayor desconfianza para la ciudadanía y diez actividades gubernamentales de intervención para mejorar la política de seguridad en el país. 
Esta propuesta metodológica tiene algunas limitantes por las características de los indicadores, puesto que, al emplear un diseño estadístico gubernamental, el contenido no es homogéneo. Como se verá posteriormente en este artículo, han ido cambiando, disminuyendo o aumentando los rubros de análisis.

Esto es posible corroborar en las gráficas 3, 4, 7 y 8. Los años que contempla la ENVIPE comprenden de 2011 a 2018, y se enfocan en la recopilación de datos sobre percepción de inseguridad, las instituciones de mayor desconfianza entre los mexicanos y el nivel de conocimiento social sobre la intervención pública. Por contraparte, la gráfica 6 muestra los resultados de 2014 a 2018 respecto a la percepción sobre la seguridad pública nacional y, la gráfica 9 evalúa de 2012 a 2018 el desempeño policiaco.

En este artículo se ha tratado de complementar la información faltante gracias al cálculo de los promedios de las tendencias de la encuesta respecto al número de habitantes en el país, con cifras de instituciones oficiales o aquellas provenientes de las asociaciones civiles, en el afán de retratar los resultados de la política de seguridad en el periodo seleccionado.

A pesar de estos límites, lo rescatable de ello es que la ENVIPE considera un muestreo representativo de la población mexicana. Es una forma de medir la efectividad de la política de seguridad proporcionando datos que puedan servir para diagnosticar desde la academia, las deficiencias sustanciales ante las barreras para la obtención y transparencia de los datos oficiales, coadyuvar desde el poder público a la toma de acciones de mejora, y reconducir aquellos rubros que se consideren endebles.

\section{Los RESUltados de LA POLÍTICA DE SEGURIDAD 2006-2018}

Históricamente la seguridad pública en el país no fue un tema prioritario hasta 2006 con el gobierno de Felipe Calderón Hinojosa. Algunas investigaciones sugieren que ante la controversia política del triunfo del Partido Acción Nacional (PAN) sobre Movimiento Regeneración Nacional (MORENA), y a las presiones norteamericanas para tomar acciones contra el narcotráfico en el país, hubo repercusiones en la reforma de la política de seguridad (Pérez, 2011; Morales, 2011; Wolf, 2011; Reyes y O'Quinn, 2013; Cansino y Molina 2010). Los cambios sustanciales se enfocaron principalmente en la Ley General del Sistema Nacional de Seguridad Pública (LGSNSP).

La LGSNSP se convirtió en un marco jurídico sin precedentes. Contempló desde las condiciones de acceso a las instituciones de seguridad, los cuerpos policiales y su profesionalización, los sistemas de acreditación de confianza, la constitución de organismos que tuvieran una base de datos con estadísticas criminales confiables y, por primera vez, se considera la participación de la ciudadanía en la pacificación del país.

La reforma policiaca fue una estrategia de gran envergadura. No existe precedente alguno como en este gobierno sobre el diseño e implementación de diagnósticos sobre sus deficiencias y fortalezas, el equipamiento, la profesionalización y el control de confianza para promover con mayor efectividad el ejercicio de los policías (Montero, 2013; Hernández, 2015; Moloeznik y Suárez, 2012).

Sobre todo, la policía federal experimentó grandes reformas. Entre otras acciones, se destinó presupuesto para recuperar el tejido social de los destinos más violentos del país, como Ciudad Juárez, se capacitó a distintos órdenes de gobierno en materia de trata de personas y, como estrategia prioritaria, se combatió el narcotráfico gracias al desmantelamiento los principales cárteles del país.

En cuanto al sexenio de Enrique Peña Nieto, la política de seguridad comenzó con estrategias conciliatorias. El sexenio transcurría y no había un estimado claro de las líneas de acción. Por tal motivo, comenzó a especularse sobre el abandono de la política de seguridad pública anterior y un "pacto" tras bambalinas con los grupos delictivos, que repercutían en el cese a la persecución de los cárteles del país.

Sin embargo, lo más representativo del sexenio fue la promulgación de la Ley de Seguridad Interior, la cual desató grandes controversias, foros de opinión pública y resistencias de la sociedad civil ante lo que denominaron una ley "inconstitucional” y "militarizante" (Medellín, 2018; Montero, 2013; De Haas, 2015). 
Mientras que se desarrollaban los debates sobre el tema, el gobierno, por su parte, dedicó esfuerzos a combatir el secuestro, que es uno de los mayores logros del sexenio. Se insistió en elaborar diagnósticos estatales sobre la situación de los cuerpos policiacos y sobre la capacitación de éstos en cuanto al uso de la fuerza, el manejo de armas y se obligó a los Estados a realizar exámenes de control de confianza.

Entre los dos sexenios esta investigación podría seguir datando los ejes de acción, pero el fin de ello es que, mediante lo enunciado hasta el momento, se pueda llevar a cabo un ejercicio de evaluación expost facto que, consiste en medir los logros de la política de seguridad pública en función de las cifras de los delitos más comunes entre la sociedad al término de su implementación.

De las cifras oficiales proporcionadas por el Secretariado Ejecutivo del Sistema Nacional de Seguridad Pública, entre 1997 al 2017, se puede observar lo siguiente: de 2006 a 2017 el homicidio doloso representó en promedio 16694 casos. La extorsión 5227 y el secuestro 1098 casos (gráfica 1). Para 2018, el promedio de homicidio se presentó en 50849 casos, el secuestro en 1559 y la extorsión en 6606 casos.

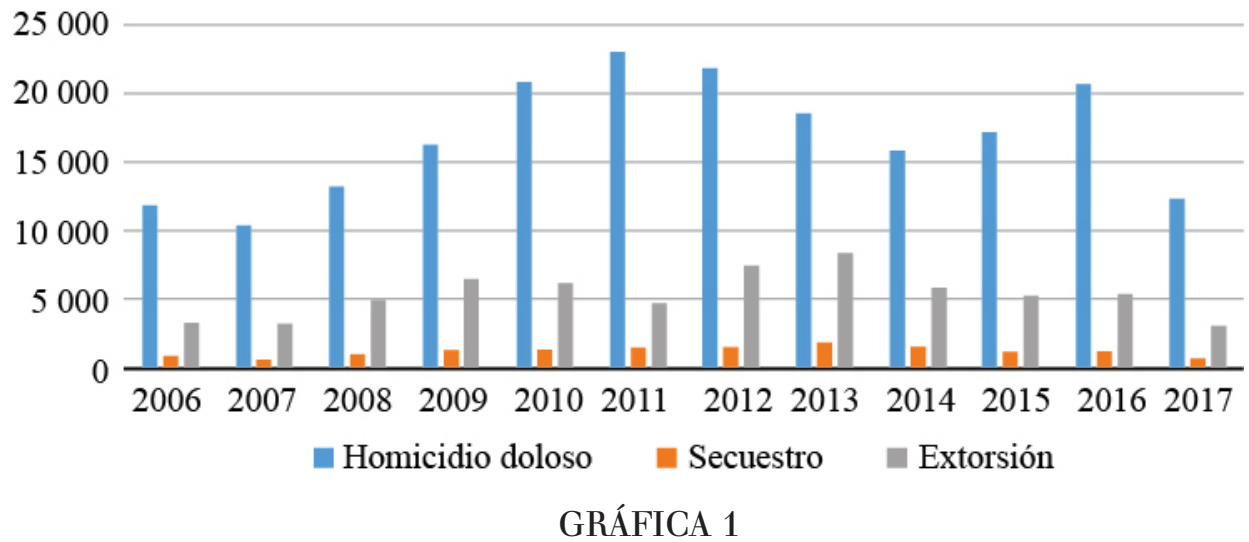

Las cifras de los delitos en México: 2006-2017

Fuente: elaboración propia a partir de los datos del Secretariado Ejecutivo del Sistema Nacional de Seguridad Pública: 1997-2017.

El contraste de las cifras de los delitos medidos en la ENVIPE permite realizar una serie de acotaciones. El crecimiento poblacional en México contabilizado desde 2010 a 2018 ha sido de 110910 millones de personas, que representa un aumento en promedio de 98200 habitantes por año. Observar el proceso de crecimiento poblacional en el país es de gran relevancia, puesto que a partir de estos datos y con lo reportado en la gráfica 2 se puede hacer un comparativo respecto al número de delitos desde 2010 a 2017.

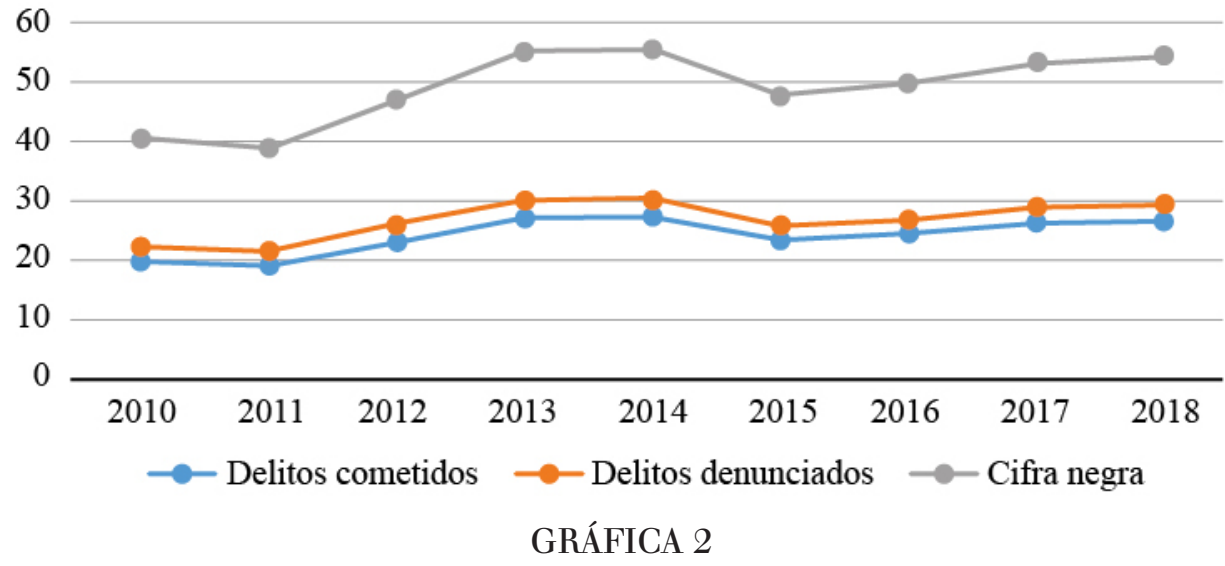

Delitos cometidos como porcentaje de la oblación total: 2010-2018 Fuente: elaboración propia a partir de los datos de la ENVIPE (2019). 
De la gráfica 2 se evidencia que, en 2010, 19.7\% de la población fue víctima de algún delito. De 2011 a 2014 las cifras incrementaron anualmente en promedio $2.7 \%$. El porcentaje más elevado se registró en 2014 con $27.1 \%$, en 2015 se redujo en $3.8 \%$ y de 2016 a 2017 las personas víctimas de la delincuencia representaron en promedio $25.1 \%$.

Respecto a la población total que denuncia en el periodo 2010 a 2017, la tasa fue de 2.55\%. Esto resulta de gran importancia, puesto que poco más de $20 \%$ de la población que anualmente es víctima de un delito, en promedio, $2 \%$ denuncia ante las instancias correspondientes.

Esto repercute en los datos reportados como cifra negra, ${ }^{[1]}$ como porcentaje de la población total, que representa $21.9 \%$ de 2010 a 2017. Aunque del periodo 2011 a 2015 se observa un aumento en promedio de cuatro puntos porcentuales, se trata de una cifra alarmante, puesto que, del número de delitos cometidos a nivel nacional, el grado de impunidad es equitativo.

\section{Percepción de inseguridad}

La interrogante que surge a partir de los datos presentados es cómo impactan en la percepción social de la seguridad en el país. La encuesta desde 2011 a 2018 preguntó a los mexicanos sobre los lugares donde se sienten más inseguros (gráfica 3). Los datos obtenidos en promedio en este periodo son los siguientes: $44.13 \%$ percibe mayor inseguridad en su colonia o localidad, $66.88 \%$ en su municipio o delegación y $72.62 \%$ en su entidad federativa. En todos los casos durante 2018 se observa el mayor incremento sobre la percepción de la inseguridad.

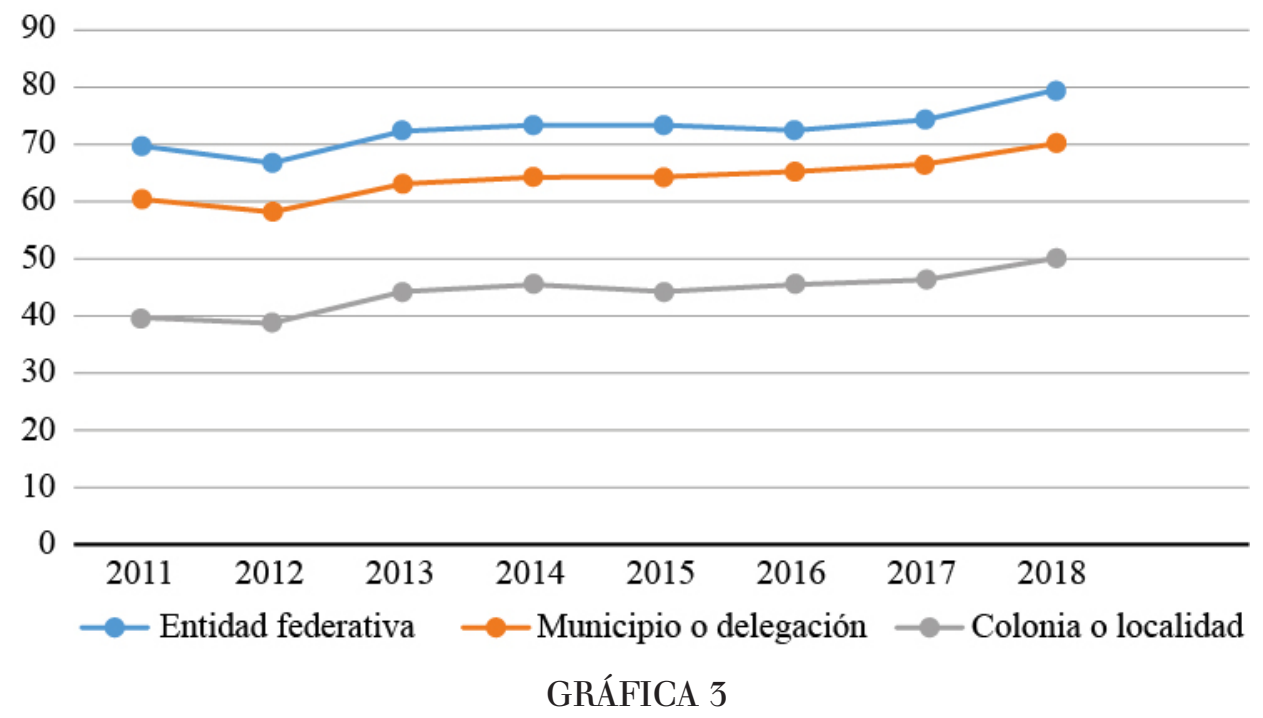

Porcentaje de la población que se siente insegura en su lugar de residencia: 2011-2018 Fuente: elaboración propia a partir de los datos de la ENVIPE (2019).

Los datos anteriores son concluyentes en el sentido de la evaluación social de la política de seguridad pública en los gobiernos subnacionales. El entorno más próximo a la ciudadanía, como es el caso de la colonia o localidad, presenta mejoras en la percepción en materia de seguridad y en el extremo, como el peor evaluado, es el gobierno estatal.

En territorio nacional, la caracterización de delitos que explican el porqué de estas variaciones es la siguiente: el promedio nacional refleja que $76.88 \%$ se percibe como posible víctima de al menos un delito, $69.95 \%$ cree que será víctima de robo o asalto en la calle o en el transporte público, $49.61 \%$ considera que experimentará lesiones por agresión física y $41.61 \%$ se asume como probable víctima de extorsión o secuestro (gráfica 4). 


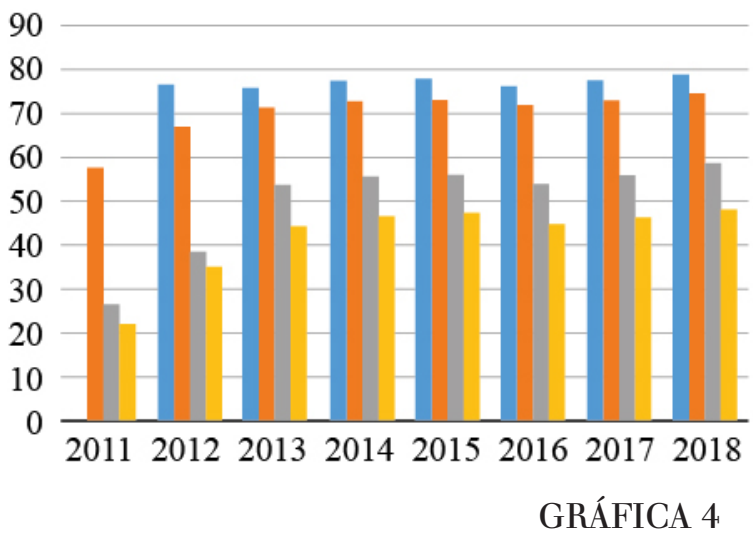

- Se percibe como posible víctima de al menos un delito

- Robo o asalto en la calle o en el transporte público

" Lesiones por agresión física

Extorsión o secuestro

Porcentaje de la población que percibe la posibilidad de ser víctima de algún delito: 2011-2018 Fuente: elaboración propia a partir de los datos de la ENVIPE (2019).

*Nota: en 2011 no se estableció como categoría de respuesta "Se percibe como posible víctima de al menos un delito", ya que se incorporó a partir del 2012.

Lo anterior repercute en que la población mexicana, al considerar la posibilidad de ser víctima de al menos un delito, ha dejado de realizar actividades que desde su imaginario influirían para convertirse en blanco de la delincuencia. El promedio nacional de 2010 a 2018, mostrado en la gráfica 5, es el siguiente: 66.6\% no permitió que sus hijos menores de edad salieran de casa, $63.82 \%$ de la población dejó de usar joyas, $52.33 \%$ no llevó a cabo actividades nocturnas, $39.45 \%$ no portó tarjeta de crédito o débito al salir y $48.47 \%$ dejó de llevar dinero en efectivo en cantidades ostentosas.

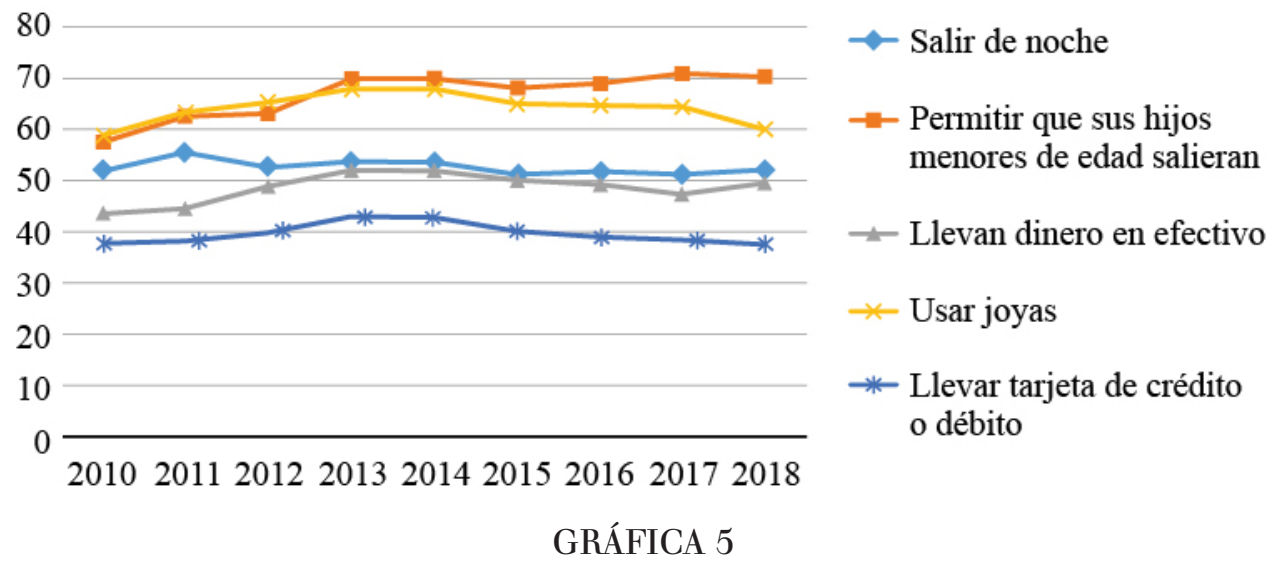

Principales actividades que la población dejó de realizar a causa de la inseguridad: 2010-2018 Fuente: elaboración propia a partir de los datos de la ENVIPE (2019).

Pese a ello, también se observa que la población ha desarrollado estrategias de sobrevivencia ante la delincuencia. Referente a esto, aunque las cifras anteriores sugieren que ha tomado precauciones, también continúa realizando actividades como viajar por carretera o acudir a actividades recreativas, entre otras, y se demuestra que ha aprendido a convivir con el entorno de violencia en los espacios públicos y privados.

\section{Confianza institucional}

La percepción de la inseguridad, analizada en el apartado anterior, incide en la valoración sobre el desempeño gubernamental en materia de seguridad pública. El promedio nacional en el periodo 2014 a 2018 (gráfica 6) reportó que 39.86\% de los encuestados consideran que la situación del país “empeorará", 31.64\% opina que "seguirá igual de mal", $19.04 \%$ cree que "mejorará" y 5.86\% reporta que "seguirá igual de bien". 
Lo contradictorio de los datos es que en 2017 aumentó 7.2\% el número de personas que consideraron que la seguridad pública empeoraría y decreció $2.6 \%$ en 2018. En contraste, para 2017, los datos en el rubro donde la población categorizó que la seguridad "seguiría igual de mal", disminuyó $2.1 \%$ y repuntó en 2018 con 2.1\%. Los primeros datos son coincidentes con lo reportado por la población al opinar que la seguridad pública mejorará, ya que se observa un decrecimiento de $2 \%$ en sus expectativas.

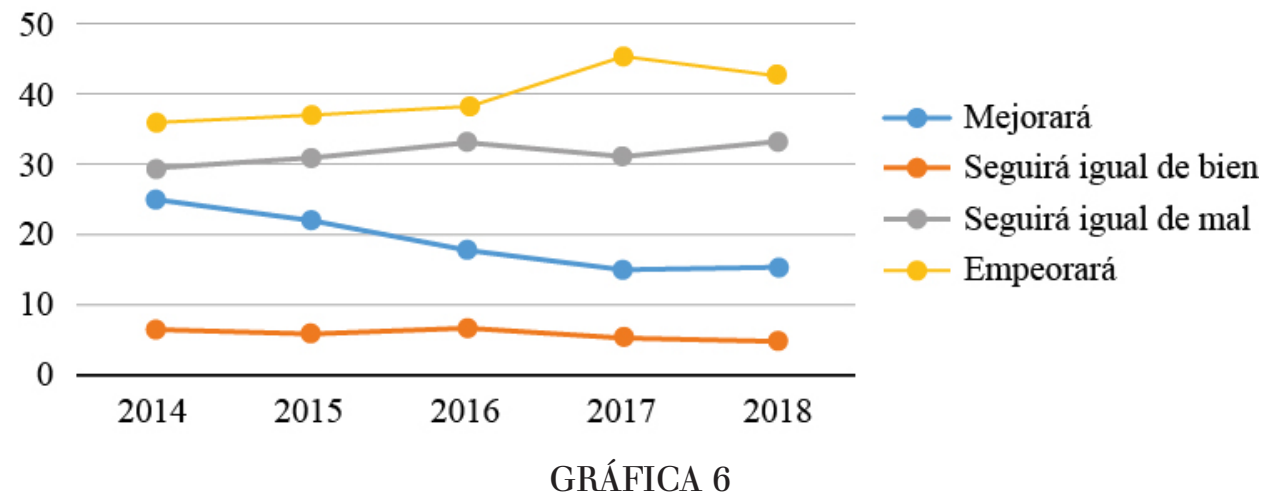

Percepción sobre la seguridad pública nacional por porcentajes: 2014-2018 Fuente: elaboración propia a partir de los datos de la ENVIPE (2019).

En cuanto al desempeño institucional de 2011 a 2018, representado en la gráfica 7, la valoración nacional consideró como las principales instituciones de confianza entre los mexicanos, la marina, el ejército y la policía federal, con 54.31, 49.37 y 19.62\%. Por contraparte, las instituciones de mayor desconfianza entre los mexicanos fueron la policía de tránsito con 24.82\%, la Policía Ministerial o Judicial con 22.23\%, el Ministerio Público y Procuradurías Estatales con 21.38\% y la Procuraduría General de la República con 16.9\%.

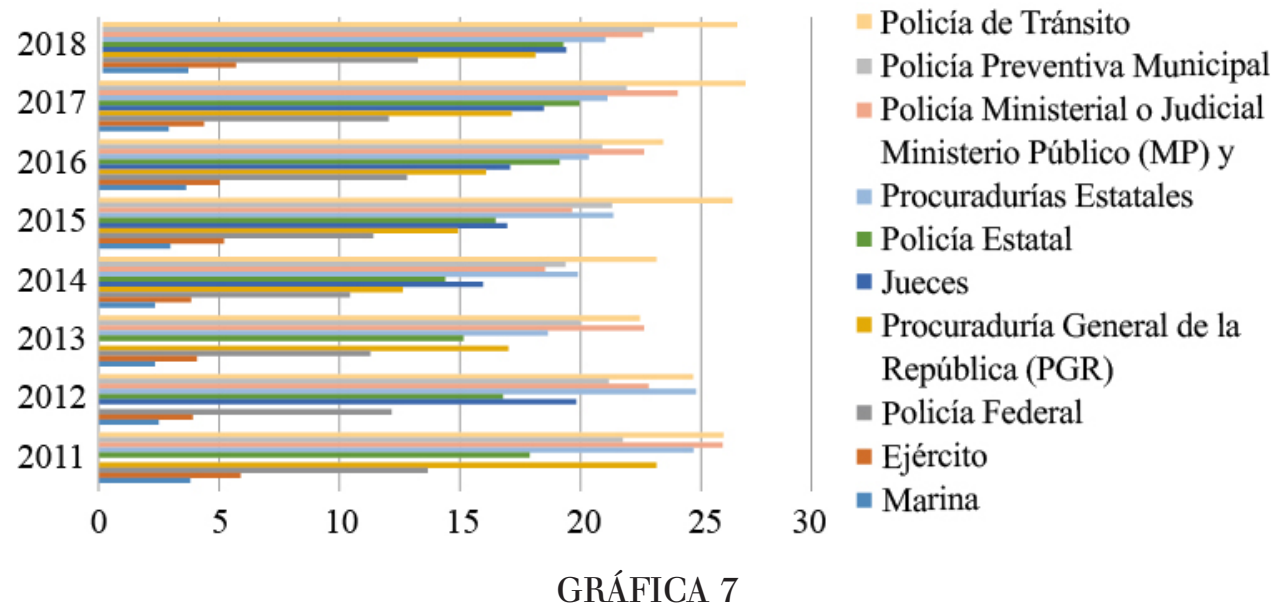

Instituciones de seguridad pública de mayor desconfianza entre los mexicanos: 2011-2018 Fuente: elaboración propia a partir de los datos de la ENVIPE (2019).

*Nota: de 2011 a 2013 no se sometió a la Procuraduría General de la República a la evaluación social, pues se incorporó a partir de 2014.

Lo anterior se relaciona con las actividades gubernamentales que el ciudadano percibe se realizan para mejorar la seguridad de su entorno. Este rubro mide el conocimiento que se tiene sobre la política pública. De las diez categorías que la ENVIPE contempla, verificables en la gráfica 8 , aquellas que son más visibles para la ciudadanía en el periodo 2011 a 2017 son las siguientes: mayor patrullaje y vigilancia con un promedio de 50.24\%, construcción y mantenimiento de parques y canchas deportivas con $47.6 \%$, mejora del alumbrado con $44.75 \%$ y, con un porcentaje menor, programas de sensibilización para que la gente denuncie con $25.81 \%$. 
Por el contrario, entre las estrategias gubernamentales menos visibles para la ciudadanía se encuentran la existencia de la policía de barrio para que coordine la seguridad con $12.11 \%$, el combate a la corrupción con $15.85 \%$ y con $17.75 \%$ la atención al desempleo.

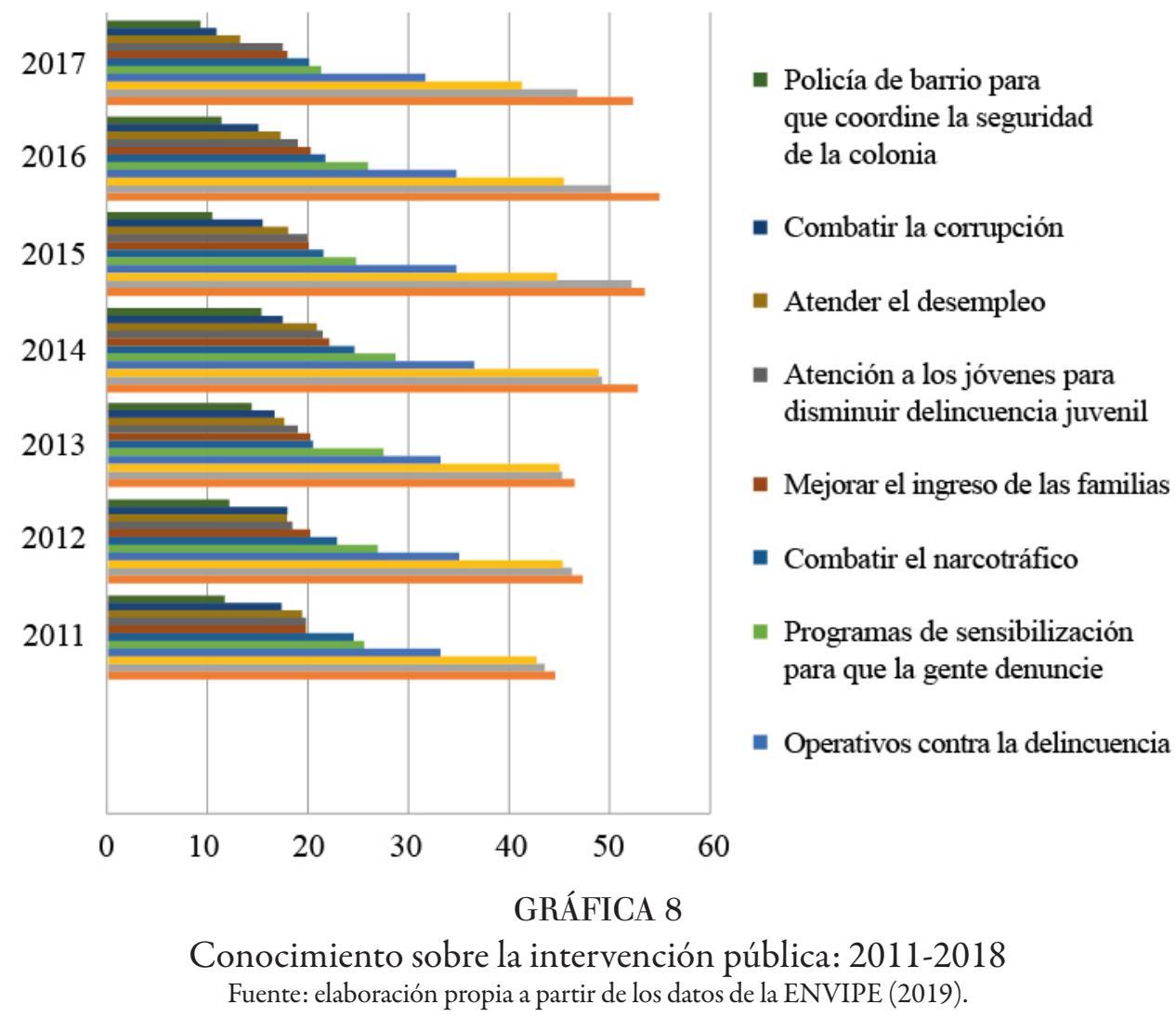

Los datos anteriores están correlacionados con la función policial (gráfica 9). En el periodo 2012-2018 se le preguntó a la población mexicana si consideraba que "la policía sí ayudaba". Los resultados reportados del promedio del periodo fueron los siguientes: la población consideró que la policía de tránsito "sí hacía algo para ayudar" con $44.16 \%$, la policía municipal con $55.21 \%$, la policía estatal con $58 \%$ y con $62.72 \%$ la policía federal.

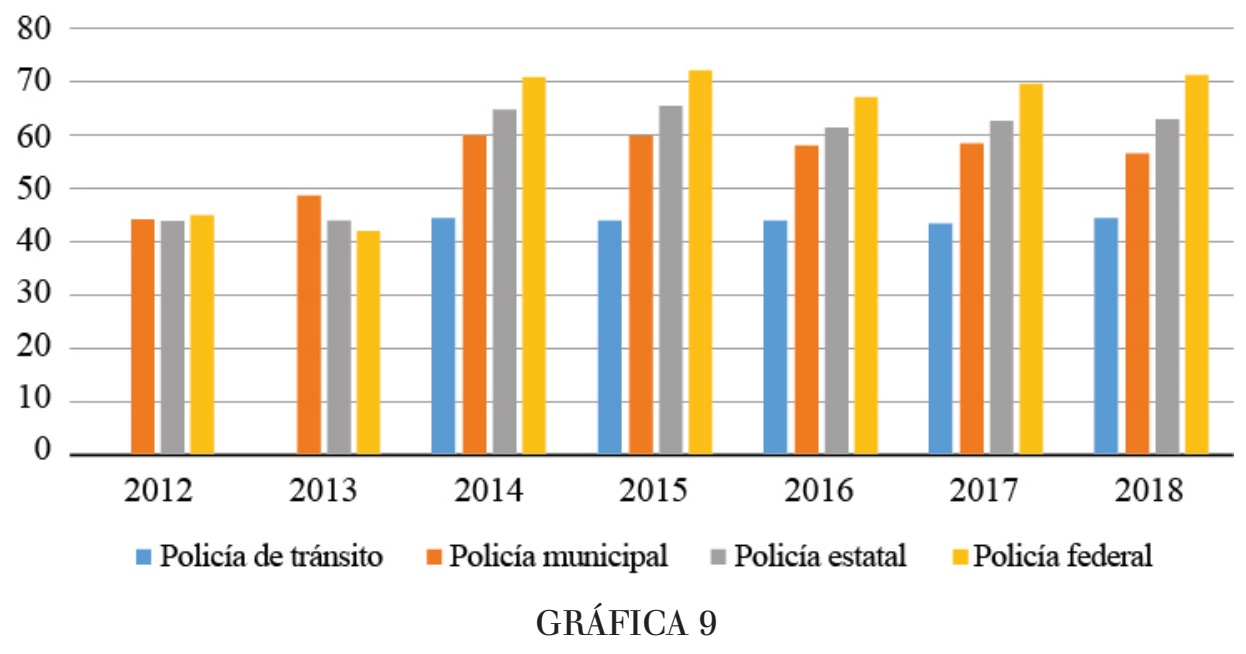

Porcentaje de personas que piensan que la policía sí ayuda: 2012-2018

Fuente: elaboración propia a partir de los datos de la ENVIPE (2019). 
Estos datos son de suma importancia porque las estrategias de seguridad en los dos sexenios se enfocaron en mejorar la función policiaca. Los mejores evaluados en promedio son la policía federal, en primer orden, y la policía estatal, en segundo. Sin embargo, la policía de tránsito está reprobada en la opinión de los mexicanos.

Estudios sobre la cultura policial demuestran que uno de los medios de ascenso promovido por los jefes inmediatos comienza con el patrullaje en las calles y el desempeño de funciones de tránsito que, a juicio de los policías, representa una forma de castigo para aquellos policías egresados de las academias (Contreras, 2017; Llanos et al., 2017; Alvarado y Silva, 2011). El entre y la mordida son dos actividades comúnmente empleadas por la policía de tránsito:

El entre es la práctica ilegal de los mandos medios a sus subalternos de exigirles dinero o servicios, sea para ascender en el escalafón, de obtener servicios considerados favorables, de fijar cuota para no ser sancionado, para ser asignado a patrullar en lugar de servicios de oficinas, etc. [...] La mordida es la extorsión del policía hacia el ciudadano, sea para cumplir el entre o porque así logra el beneficio económico y su conducta la racionaliza de varias maneras: porque el ciudadano es el que me corrompe, porque si yo detengo a un delincuente, después lo sueltan, etc. (Orellana, 2017: 99-100).

Esto incide negativamente en el respeto a la autoridad y en la confianza institucional, ya que al ser policías de proximidad, el ciudadano prefiere participar en estos actos de corrupción, que ser sancionados por multas o convertirse en víctimas de abuso de autoridad.

\section{Prospectiva del delito: ¿Qué se Puede esperar Para los Primeros años del sexenio actual?}

A modo de conclusión, los resultados del análisis de la ENVIPE demuestran que los delitos respecto al número total de habitantes en el país han evolucionado en cifras de aproximadamente $20 \%$ en promedio, lo cual estadísticamente no resulta tan elevado en comparación con la valoración social de la inseguridad. De la sociedad mexicana, 39.86\% considera que la situación del país empeorará. Además, entre más lejano sea el espacio de residencia, más incrementa la percepción negativa de la inseguridad. Las entidades federativas son las que obtienen el peor porcentaje con $72.62 \%$ de la valoración.

De acuerdo con el número de delitos que han sido reportados en el periodo de estudio, la cifra casi se cuadruplica en cuanto a la percepción de "ser víctima de al menos un delito" con 76.88\%. En lo referente al robo, la percepción casi se triplica con $69.95 \%$. Respecto a lesiones y agresión física, la cifra se duplica en $49.6 \%$ y, en lo relativo al secuestro y extorsión, la percepción se duplica en $41.61 \%$.

Entre los hallazgos más impactantes se encuentra lo reportado en la cifra negra, pues es prácticamente equivalente al número de delitos ocurridos en el país. Esto se explica con los datos sobre la confianza institucional y el desempeño policiaco que han repercutido en el hecho de que la sociedad considere, de acuerdo con la encuesta, una "pérdida de tiempo" denunciar porque percibe actos de corrupción o escasa capacitación en las averiguaciones previas e investigación para el levantamiento de pruebas.

A pesar de estas subjetividades colectivas, la evidencia muestra un incremento del presupuesto asignado en materia de seguridad desde 2006. En ese año representó 139353 millones de pesos; en 2012, 252550 millones de pesos; en 2016, 277308 millones de pesos, de los cuales, la Secretaría de la Defensa Nacional (SEDENA) obtuvo los mayores incrementos. En 2006 tuvo un presupuesto inicial de 40515 millones de pesos y en 2012 33846 millones de pesos. La Procuraduría General de la República (PGR), de 13305 millones de pesos que le fueron asignados en 2006, en 2012 tuvo un presupuesto de 3303 millones de pesos más. Al poder Judicial de la Federación (PJF) se le asignaron 35730 millones de pesos y hasta 2012 reflejó ingresos superiores de 22252 (mdp) (Secretaría de Hacienda y Crédito Público, 2006, 2012).

A partir de los datos expuestos, se concluye que las mayores inversiones monetarias y en capacitación se dedicaron a los ministerios públicos y a los cuerpos de seguridad. Sin embargo, la población percibió como parte de 
la intervención gubernamental un mayor desplegado de policías, vigilancia y patrullaje, rehabilitación de canchas y mejoramiento del alumbrado público y, en menor medida, las acciones concretas contra la delincuencia, profesionalización de los ministerios y procuradurías estatales y resolución de sentencias.

Esto resulta contradictorio si se aclara que, de acuerdo con la Cuenta de la Hacienda Pública Federal de 2006 a 2016, el Fondo de Aportaciones para la Seguridad Pública para Estados y Municipios (FASP) tuvo tendencia a disminuir y ha representado la menor transferencia monetaria a nivel federal. Esto es, en 2006, comenzó con un presupuesto de 7506 millones de pesos; en 2012 obtuvo 8464 millones de pesos y en 2016 disminuyó a 7000 millones de pesos (Secretaría de Hacienda y Crédito Público, 2006, 2012, 2016).

Por otra parte, las acciones que sí repercutieron en la valoración positiva fueron los resultados obtenidos respecto a la policía federal, el ejército y la marina que formaron parte de la confianza institucional reportada por la sociedad mexicana. Principalmente, se debe a las estrategias entre los dos sexenios que emplearon en operativos a estas instituciones en contra del crimen organizado.

Por lo tanto, la pregunta de investigación a partir de los datos anteriores corrobora que los delitos en el país son menores a lo estimado socialmente y, por consecuencia, no corresponden con el incremento exorbitante de la valoración negativa de la seguridad pública y de las actividades cotidianas que la población mexicana ha dejado de hacer para evitar ser víctimas del delito. Esto se considera de acuerdo con los especialistas del tema, como trastorno de estrés postraumático de la violencia (Moreno y Villamil, 2003; Martín y Paúl, 2004; Rincón, 2004), el cual consiste en que la población, aunque no haya sido víctima de la delincuencia, por la difusión de los medios de comunicación y la socialización del temor entre las personas se crea la idea de un entorno de mayor inseguridad, además del desencanto político que genera que la valoración social en casi cualquier rubro sea reprobatoria.

Respecto a la hipótesis de este trabajo, ésta se comprueba, puesto que a pesar de haber un repunte en el número de homicidios en 2011 con 22852 casos y en 2012 con 21736 casos; por el contrario, en 2014 y 2017 disminuyeron con 15660 y 12155 casos. En particular, la extorsión y el secuestro se mantuvieron en cifras bajas, como se observó en la gráfica 1.

Con la entrada del gobierno actual, el Plan Nacional de Paz y Seguridad (2018-2024) contempla como objetivos programáticos la reducción de los índices delictivos, la tipificación de la corrupción como delito grave y la revisión de los patrimonios personales. De manera complementaria, se introducen acciones para atacar de raíz el problema de la delincuencia como lo son la reducción de la pobreza, la marginación y la desintegración familiar y social, las cuales se acompañan de programas de desarrollo y bienestar sectoriales. Esto puede incidir en los resultados de la ENVIPE del año siguiente, ya que en los años analizados hasta la última actualización de la encuesta la población no percibe mejoras en el desempleo, el ingreso de las familias y, en específico, en el combate a la corrupción.

En cuanto a acciones concretas de seguridad pública y de los cuerpos policiacos se reorientará el papel de las fuerzas armadas, se creó la Guardia Nacional (actualmente en funciones), se establecerán coordinaciones regionales para detectar las zonas con mayores índices de violencia, además de las reformas a la Ley Orgánica de la Administración Pública Federal (LOAPF) que posibilita la creación de la Secretaría de Seguridad y Protección Ciudadana (SSPC) como responsable de la seguridad pública nacional (Observatorio Nacional Ciudadano, 2019). No obstante, para legitimar estas acciones el gobierno federal ha iniciado una campaña de desprestigio oficial y el desmantelamiento de la policía federal para la conformación de la Guardia Nacional, lo cual ha ocasionado disputas entre los policías y el gobierno federal.

La tendencia respecto al delito se debería mantener en los números que en promedio se han evidenciado en esta investigación. Sin embargo, en lo que va de enero a junio de 2019 se ha observado un incremento de los delitos con respecto a sexenios anteriores. Según las cifras del Secretariado Ejecutivo del Sistema Nacional de Seguridad Pública (2019), la incidencia delictiva en este periodo es de 1004191 casos que, de acuerdo con los datos de la población mexicana reportados por el Banco Mundial, México contaba en 2017 con 129200 millones de habitantes. Es decir, los delitos acumulados en el sexenio como porcentaje de la población total 
representa $0.77 \%$ que, a simple vista, no parece alarmante. No obstante, en otros datos reportados por el Secretariado Ejecutivo, 17010 personas fueron asesinadas en México en el primer semestre del gobierno actual que, de acuerdo con sus estimados, corresponde a poco más de 93 individuos por día.

Los homicidios dolosos reportados en junio por la Secretaría de Seguridad Federal, en mayo representaron 2903 casos convirtiéndose en el mes más violento en comparación con el sexenio de Peña Nieto. Entre otras cifras reportadas por Semáforo Delictivo (2019), hasta el corte de agosto del año que transcurre, se registraron 2484 homicidios, 102 secuestros, 787 extorsiones, 6518 casos de narcomenudeo, 15007 robos a vehículos, 7158 robos a casas, 9305 robos a negocios, 13232 lesiones a causa de la violencia, 1487 violaciones, 18604 casos de violencia familiar y 88 feminicidios. En general, de seguir esta tendencia, 2019 podría convertirse en el año más violento del país.

Sin embargo, entre los avances de este sexenio, se encuentra el incremento del presupuesto en 2019 para combate al crimen de 101000 millones de pesos que corresponde a $0.41 \%$ del Producto Interno Bruto (PIB), además de 104755 millones de pesos para el mejoramiento de la justicia; una cifra que representa $0.42 \%$ del PIB y un retroceso de $13.4 \%$ respecto al presupuesto de 2018 (Observatorio Nacional Ciudadano, 2019), lo cual es notablemente diferenciador de los sexenios anteriores.

El impacto de este gasto, como fue posible analizar, no repercute en el mejoramiento de la percepción social, incluso las cifras de desplazamientos forzados a causa de la violencia han ido en aumento. De acuerdo con las cifras del Banco Mundial (2019), en México de 2009 a 2018 ha habido en promedio 217700 personas que han abandonado sus lugares de residencia a causa de la violencia, la descomposición social y la corrupción de las instituciones. Los años con mayores desplazamientos forzados se ubican en 2016, 2017 y 2018 con 311000 , 45000 y 338000 casos.

Como conclusión, la evaluación externa y ex post facto ha reportado que la capacitación de policías de proximidad, los ministerios y procuradurías estatales no está siendo efectiva porque las condiciones de los policías no están atendidas en equipamiento y en el control de confianza. Por tanto, la sociedad mexicana no tiene expectativas a futuro sobre una mejoría de la seguridad pública. Para los gobiernos estatales y el federal, esto debería ser tema de interés al ser evidente que su población beneficiaria no encuentra satisfechas sus demandas en materia de seguridad ni sobre el desempeño del gobierno.

\section{ReFERENCIAS}

Alvarado, A. (2010). Inseguridad pública, participación ciudadana y gobernanza. La ciudad de México en la última década. Estudios Sociológicos, 28(84), 941-963.

Alvarado, A. y Silva, C. (2011). Relaciones de autoridad y abuso policial en la Ciudad de México. Revista Mexicana de Sociologia, 73(3), 445-473.

Banco Mundial. (2019). Disponible en https://datos.bancomundial.org/ indicator/VC.IDP.TOCV ?locations $=\mathrm{MX}$

Becerra, M. y Trujano, P. (2011). Percepción de inseguridad pública y justificación de la violencia de estado en un grupo de habitantes del Estado de México: análisis de argumentos. Acta Colombiana de Psicología, 14(2), $35-43$.

Bustelo, M. (2001). La evaluación de las politicas públicas de igualdad de género de los gobiernos central y autonómicos en España: 1995-1999 (tesis doctoral). Madrid: Universidad Complutense de Madrid.

Cansino, C. y Molina, G. (2010). La guerra al narco y otras mentiras. Argumentos a favor de la legalización de las drogas en México. IUS. Revista del Instituto de Ciencias Juridicas de Puebla A.C., 4(26), 304-306. 
Cardozo, M. (2013). Políticas públicas: los debates de su análisis y evaluación. Andamios. Revista de Investigación Social, 10(21), 39-59.

Contreras, O. (2017). Institución policial, violencia y cultura del terror en Tijuana. Revista Mexicana de Sociologia, 79(4), 697-721.

Chávez, S. y Esparza, O. (2017). Validación de una escala de percepción de riesgo en jóvenes mexicanos. Enseñanza e Investigación en Psicología, 22(1), 110-117.

De Haas, M. (2015). Comparación entre el marco constitucional de Seguridad Nacional e Interior de México y Argentina. URVIO. Revista Latinoamericana de Estudios de Seguridad, 17, 97-108.

Dzib, N., Campos, M., Novelo, V. y Pérez, D. (2016). Percepción de riesgo y clima de seguridad en operadores de transporte en Mérida, Yucatán. Acta de Investigación Psicológica, 6(1), 2277-2285.

ENVIPE (Encuesta Nacional de Victimización y Percepción sobre Seguridad Pública). (2019). Disponible en: https://www.inegi.org.mx/programas/envipe/2019/default .html\#Tabulados

García, C. (2012). La estructura de la percepción de la inseguridad pública. Liberabit. Revista de Psicología, 18(1), $37-44$.

Gómez, R. (2012). La percepción sobre la seguridad pública como determinante del voto en la opinión pública en México. Revista Mexicana de Opinión Pública, 12, 31-53.

Hernández, G. (2015). Análisis de la política de seguridad en México 2006-2012. Política y Cultura, 44, 159-187

Laca, F. y Navarro, F. (2013). La percepción de la violencia en México en relación con el bienestar subjetivo y social. Anuario de Psicología, 43(3), 323-334.

Llanos, L., Rivas, L., Lambarry, F. y Trujillo, M. (2017). La eficacia de la policía en México: un enfoque cualitativo. Polis. Investigación y Análisis Sociopolítico y Psicosocial, 13(2), 107-138.

Manero, R. y Villamil, R. (2003). El correlato de la violencia en el síndrome de estrés postraumático. El Cotidiano, 19(121), 6-25.

Martín, J.y Paúl, J. (2004). Trastorno por estrés postraumático en víctimas de situaciones traumáticas. Psicothema, $16(1), 45-49$

Medellín, L. (2018). La Ley de Seguridad Interior en México: entre la razón de Estado y la justificación de los derechos humanos. URVIO. Revista Latinoamericana de Estudios de Seguridad, 23, 142-159.

Moloeznik, M.y Suárez, M. (2012). El proceso de militarización de la seguridad pública en México (2006-2010). Frontera Norte, 24(48), 121-144.

Montero, J. (2013). El concepto de seguridad en el nuevo paradigma de la normatividad mexicana. Región y Sociedad, 25(58), 203-238.

Morales, C. (2011). La guerra contra el narcotráfico en México. Debilidad del estado, orden local y fracaso de una estrategia. Aposta. Revista de Ciencias Sociales, 50, 1-35.

Observatorio Nacional Ciudadano. (2019). Gasto público en seguridad. Una mirada a la ruta de los recursos. Disponible en http://onc.org.mx/wp-content/uploads/2019/06/ReporteSeguridad_CIEP_ONC_VF.pdf

Olvera, T. (2013). Evaluación de politicas públicas: estudio de caso del Programa Escuelas de Calidad en el Estado de Hidalgo, México, 2001-2005 (tesis doctoral). Madrid: Universidad Complutense de Madrid.

Orellana, O. (2017). Seguridad pública: profesionalización de los policias. México: Porrúa.

Pérez, J. (2011). La guerra contra el narcotráfico: ¿una guerra perdida? Espacios Públicos, 14(30), 211-230.

Rebolledo, D. (2015). La violencia como limitante para el desarrollo y el crecimiento económico en el estado de Guerrero. Revista Mexicana de Ciencias Agricolas, 1, 385-390. 
Reyes, R. y O'Quinn, J. (2013). La comunicación gubernamental de la guerra contra el narcotráfico en México. Espacios Públicos, 16(36), 55-75.

Rincón, P. (2004). Trastorno de estrés postraumático en mujeres víctimas de violencia doméstica: evaluación de programas de intervención (tesis doctoral). Madrid: Universidad Complutense de Madrid.

Rodríguez, D. (2012). El sentimiento de inseguridad y su repercusión social y política. Espiral, 19(55), 201-208.

Ruiz, J. y Turcios, L. (2009). Percepción de seguridad, victimización y cultura ciudadana: sus relaciones en cinco contextos iberoamericanos. Pensamiento Psicológico, 6(13), 193-202.

Salazar, C. (2009). La evaluación y el análisis de políticas públicas. Revista Opera, 9, 23-51.

Sánchez, V.y Aguilar, S. (2016). Percepción de los turistas mexicanos sobre la imagen turística de Mazatlán ante un escenario de inseguridad. Teoría y Praxis, 20, 155-186.

Scriven, M. (1967). The methodology of evaluation. In R. Tyler, R. Gagné y M. Scriven, Perspectives on Curriculum Evaluation (pp. 39-83). Chicago: Rand McNally.

Secretaría de Hacienda y Crédito Público. (2006). Disponible en: https://www.apartados.hacienda.gob.mx/ contabilidad/documen tos/informe_cuenta/2006/index.html

Secretaría de Hacienda y Crédito Público. (2012). Disponible en: https://www.apartados.hacienda.gob.mx/ contabilidad/documentos/informe_cuenta/2012/docs/i01/i01d01.pdf

Secretaría de Hacienda y Crédito Público. (2016). Disponible en: https://www.cuentapublica.hacienda.gob. $\mathrm{mx} / \mathrm{es} / \mathrm{CP} / 2016$

Secretariado Ejecutivo del Sistema Nacional de Seguridad Pública. (2019). Disponible en http://secretariadoejecutivo.gob.mx/docs/pdfs/cifras\%20de\%20homicidio\%20doloso\%20secuestro\%20etc/HDSECEXTRV_062017.pdf

Semáforo Delictivo. (2019). Disponible en http://www.semaforo.mx/ content/semaforo-de-cifra-negra

Soria, R. (2017). Impacto de la violencia e inseguridad en la competitividad de los estados mexicanos. Economía, Sociedad y Territorio, 17(54), 279-307.

Soria, R. (2018). Una estimación del costo de la inseguridad y la delincuencia en México: análisis comparativo a nivel de las entidades federativas. Gestión y política pública, 27(1), 111-147.

Tapia, J. (2013). La inseguridad pública: causas y consecuencias. El Cotidiano, 180, 103-112.

Wolf, S. (2011). La guerra de México contra el narcotráfico y la Iniciativa Mérida: piedras angulares en la búsqueda de legitimidad. Foro Internacional, 51(4), 669-714.

Notas

[1] La importancia de reflejar el cálculo de la cifra negra en la gráfica 2 es un dato que permite comparar los grados de impunidad en el país a causa del desempeño de las instituciones de seguridad pública - desde los policías hasta la impartición de justicia-, ya que se refiere a los delitos no denunciados, delitos sin averiguación previa o no especificados en cuanto a la denuncia o la averiguación previa. El cálculo se efectúa "como la razón de los delitos no denunciados más los delitos denunciados sin averiguación previa o carpeta de investigación más aquellos en los cuales no fue especificado si se denunció o si se inició averiguación previa o carpeta de investigación, entre el total de delitos por cien.” (ENVIPE, 2019). De acuerdo con la asociación Semáforo Delictivo (2019), las estimaciones de la cifra negra en México son las siguientes:

Denunciado Real

1 extorsión 6 extorsiones

1 violación 4 a 5 violaciones

1 secuestro 3 a 4 secuestros

1 lesión 2 a 3 lesiones

1 robo a casa 2 a 3 robos a casa 
1 robo a negocio

10 robos de autos

100 homicidios
2 a 3 robos a negocio

11 robos de autos

102 homicidios

Con base en las encuestas aplicadas a nivel nacional, se han identificado algunas de las razones de las variaciones en la cifra negra. Las víctimas de la delincuencia han señalado siete causas por las que no acuden a las instancias correspondientes a denunciar: "actitud hostil de la autoridad", "miedo al agresor", "trámites largos y difíciles", "no tenía pruebas", "delito de poca importancia", "desconfianza a la autoridad" y "pérdida de tiempo" (Semáforo Delictivo, 2019). La ENVIPE (2019) reporta que la cifra negra es atribuible a tres aspectos: "a la autoridad", "a causas no asociadas a la autoridad" o a "fallos del ministerio público".

\section{BY-NC-ND}

